\title{
Dynamics of a delayed SEIQ epidemic model
}

\author{
Wanjun $\mathrm{Xia}^{1 *}$, Soumen Kundu² and Sarit Maitra ${ }^{2}$
}

\author{
"Correspondence: \\ xiawj0982@sina.com \\ 1School of Management Science \\ and Engineering, Anhui University \\ of Finance and Economics, Bengbu, \\ China \\ Full list of author information is \\ available at the end of the article
}

\begin{abstract}
In this work we consider an epidemic model that contains four species susceptible, exposed, infected and quarantined. With this model, first we find a feasible region which is invariant and where the solutions of our model are positive. Then the persistence of the model and sufficient conditions associated with extinction of infection population are discussed. To show that the system is locally asymptotically stable, a Lyapunov functional is constructed. After that, taking the delay as the key parameter, the conditions for local stability and Hopf bifurcation are derived. Further, we estimate the properties for the direction of the Hopf bifurcation and stability of the periodic solutions. Finally, some numerical simulations are presented to support our analytical results.
\end{abstract}

Keywords: SEIQ model; Delay; Boundedness; Lyapunov functional; Persistence; Hopf bifurcation; Periodic solution

\section{Introduction}

Since the principles to the mathematical model of epidemics, as the susceptible-infectedsusceptible (SIS) model and the susceptible-infected-removed (SIR) model, were presented in [1,2], the mathematical investigation of disease transmission has developed quickly. As is well known, many epidemic diseases such as HIV/AIDS [3], H1N1 [4], H5N1 [5] and SARS [6], are harmful to individual health and to the stability of our society. It is an increasingly urgent issue to control the prevalence of epidemic diseases. Mathematical epidemiology, which describes the prevalence of epidemic diseases by building and analyzing mathematical models, has been one of the major areas of biology. In recent years, mathematical models have become one of the important tools in the investigation of the prevalence and control of epidemic diseases since the pioneering work of Kermack and Mckendrick [7, 8]. For example, the SIRS (Susceptible-Infectious-RecoveredSusceptible) epidemic model [9-12], the SEIS (Susceptible-Exposed-Infectious-Susceptible) epidemic model [13-16], SEIR (Susceptible-Exposed-Infectious-Recovered) epidemic model [17-20], the SEIRS (Susceptible-Exposed-Infectious-Recovered-Susceptible) epidemic model [21-25] and epidemic models with vaccination [26-28].

In real world, some people can be quarantined once they are found to have been infected with epidemic diseases in the exposed state or the infectious state. Based on this consider-

(c) The Author(s) 2018. This article is distributed under the terms of the Creative Commons Attribution 4.0 International License (http://creativecommons.org/licenses/by/4.0/), which permits unrestricted use, distribution, and reproduction in any medium, provided you give appropriate credit to the original author(s) and the source, provide a link to the Creative Commons license, and indicate if changes were made. 
Table 1 Parameters and their meanings in this paper

\begin{tabular}{ll}
\hline Parameter & Description \\
\hline$A$ & the constant recruitment rate of the population \\
$\beta$ & The infection rate of the susceptible population \\
$c$ & The natural mortality rate of all populations \\
$\varepsilon$ & The rate that the infected recovers and comes into the susceptible class \\
$\alpha$ & The rate at which some exposed people become infective \\
$\sigma_{1}$ & The mortality rate of the infected and quarantined population due to disease \\
$\sigma_{2}$ & The quarantined rate of the exposed population \\
$\gamma_{1}$ & The quarantined rate of the infected population \\
$\gamma_{2}$ & The recovery rate of the exposed population \\
$\gamma_{3}$ & The recovery rate of the infected population \\
\hline
\end{tabular}

ation, recently, Chen et al. [29] proposed the following epidemic model with quarantine:

$$
\left\{\begin{array}{l}
\frac{d S(t)}{d t}=A-\beta S(t) I(t)-\mu S(t)+c I(t), \\
\frac{d E(t)}{d t}=\beta S(t) I(t)-\left(\mu+\varepsilon+\sigma_{1}+\gamma_{1}\right) E(t), \\
\frac{d I(t)}{d t}=\varepsilon E(t)-\left(\mu+\alpha+c+\sigma_{2}+\gamma_{2}\right) I(t), \\
\frac{d Q(t)}{d t}=\sigma_{1} E(t)+\sigma_{2} I(t)-\left(\mu+\alpha+\gamma_{3}\right) Q(t),
\end{array}\right.
$$

where $S(t), E(t), I(t)$ and $Q(t)$ are the numbers of the susceptible, the exposed, the infected and the quarantined individuals at time $t$, respectively. The meanings of the parameters are listed in Table 1.

With this model they [29] investigated the local and the global stability of system (1), and they also estimated the domain of attraction of system (1).

As stated in [30], most infectious diseases evolve by infection, and then there appear some symptoms needing a period of time (namely the incubation period). Therefore, if an epidemic model considers time delay, then it is more consistent with the actual situation [11, 12, 14, 22, 23]. Compared with ordinary differential equations, delay differential equations exhibit more complicated dynamics, such as the loss of stability, oscillations and periodic solutions. Recently, there appeared some work about epidemic models with time delay. In [31], Bai and Wu studied the traveling waves of a delayed SIR epidemic model with nonlinear incidence. In [32], Liu et al. investigated the asymptotic properties of a stochastic delayed SIR epidemic model with temporary immunity. Liu et al. [33, 34] analyzed the Hopf bifurcation of different SIRS epidemic model with time delay. Liu et al. [35] studied the global attractiveness and persistence of a delayed SIRS epidemic model on a scale-free network. Liu and Wang [36] investigated the Hopf bifurcation of an SIRS epidemic model with delays and stage structure. Sharma et al. [37, 38] analyzed the impact of time delay on the dynamics of different SEIR epidemic model. Jiang et al. [39] considered an SEIRS system with two delays and the general nonlinear incidence rate and dealt with the global Hopf bifurcation and permanence of the model. Liu and Wang [40] studied the Hopf bifurcation of an SIQR epidemic model with two delays and a nonlinear incidence rate. Obviously, most of the epidemic models with time delay above neglect the effect of quarantine. Based on this consideration and motivated by the work above, we incorporate 
the latent delay into system (1) and study the following delayed system:

$$
\left\{\begin{array}{l}
\frac{d S(t)}{d t}=A-\beta S(t) I(t)-\mu S(t)+c I(t), \\
\frac{d E(t)}{d t}=\beta S(t) I(t)-\left(\mu+\sigma_{1}+\gamma_{1}\right) E(t)-\varepsilon E(t-\tau), \\
\frac{d I(t)}{d t}=\varepsilon E(t-\tau)-\left(\mu+\alpha+c+\sigma_{2}+\gamma_{2}\right) I(t), \\
\frac{d Q(t)}{d t}=\sigma_{1} E(t)+\sigma_{2} I(t)-\left(\mu+\alpha+\gamma_{3}\right) Q(t),
\end{array}\right.
$$

subject to the initial conditions

$$
\begin{aligned}
& S(\theta)=\phi_{1}(\theta)>0, \\
& E(\theta)=\phi_{2}(\theta)>0, \\
& I(\theta)=\phi_{3}(\theta)>0, \\
& Q(\theta)=\phi_{4}(\theta)>0, \quad \theta \in[-\tau, 0), \phi_{i}(0)>0, i=1,2,3,4,
\end{aligned}
$$

where the meanings of the parameters are given in Table 1 and they are assumed to be positive and $\tau$ is the latent delay of the disease.

The organization of the paper is as follows. In the next section, it is shown that the solution of (2) is positive and bounded in a feasible region $\bar{R}$, which is invariant. Also, the persistence of the proposed model and some sufficient conditions associated with extinction of infective population are discussed. In Sect. 3, the condition for local asymptotical stability is examined by constructing a suitable Lyapunov functional. By taking the latent delay $\tau$ as the bifurcation parameter, the conditions for the occurrence of Hopf bifurcation are derived in Sect. 4. Further, the direction of Hopf bifurcation and the stability of the periodic solution are examined in Sect. 5. Some numerical results are carried out for our expository results in Sect. 6. Finally, the paper ends with the conclusion of the work.

\section{The boundedness, persistence and extinction of infected population}

\subsection{The boundedness}

In this section we shall discuss about the positivity and boundedness of solution of system (2).

For this purpose, we assume the function $V$ to be

$$
V(t)=S(t)+E(t)+I(t)+Q(t) .
$$

Taking the derivative of (4) and using (2) we get

$$
\dot{V}(t)=A-\mu S(t)-\left(\mu+\gamma_{1}\right) E(t)-\left(\mu+\alpha+\gamma_{2}\right) I(t)-\left(\mu+\alpha+\gamma_{3}\right) Q(t)
$$

where $S(t)>0$ and $E(t), I(t), Q(t) \geq 0$.

If $E(t)=0, I(t)=0$ and $Q(t)=0$ from (5) we get

$$
\lim _{t \rightarrow \infty} \sup V(t) \leq \frac{A}{\mu} .
$$


Also, if $V(t)>\frac{A}{\mu}$ then $\dot{V}(t)<0$. Therefore, we get $0<V \leq \frac{A}{\mu}$, i.e., we get a feasible region $\bar{R}$ :

$$
\bar{R}=\left\{(S(t), E(t), I(t), Q(t)) \in R^{4}: 0<S(t)+E(t)+I(t)+Q(t) \leq \frac{A}{\mu}\right\} .
$$

Thus we see that the solution of system (2) is bounded and independent of the initial condition. So the feasible region $\bar{R}$ is an invariant set. Also, as $A>0, \mu>0, \frac{A}{\mu}>0$, i.e., the feasible region $\bar{R}$ is positive.

Hence all solutions of (2) will enter the field $\bar{R}$ and will remain in $\bar{R}$.

\subsection{The persistence and extinction of infection species}

In this section, we will consider the ultimate state of infection, that is, the disease will be either persistent or extinct ultimately.

Since the variable $Q$ does not appear explicitly in the first three equations in system (2), we need only to consider the dynamics of a subsystem consisting of the first three equations in system (2). We have

$$
\begin{aligned}
& \frac{d S(t)}{d t}=A-\beta S(t) I(t)-\mu S(t)+c I(t), \\
& \frac{d E(t)}{d t}=\beta S(t) I(t)-\left(\mu+\sigma_{1}+\gamma_{1}\right) E(t)-\varepsilon E(t-\tau), \\
& \frac{d I(t)}{d t}=\varepsilon E(t-\tau)-\left(\mu+\alpha+c+\sigma_{2}+\gamma_{2}\right) I(t),
\end{aligned}
$$

From the first equation in system (7), we have $\frac{d s}{d t} \leq \mu\left(\frac{A}{\mu}-S\right)$; it implies that $\lim _{t \rightarrow \infty} \sup S(t) \leq \frac{A}{\mu}$; therefore, the set $\omega=\left\{(S, E, I) \in R_{+}^{3}: S \leq \frac{A}{\mu}\right\}$ is positively invariant under system (7). Thus, we only consider the dynamical behavior of system (7) on the set $\omega$.

When $R_{0}<1$, define the function

$$
V_{1}=\rho E+I,
$$

where $\rho \in\left(\frac{1}{\varepsilon}, \frac{\mu+\alpha+c+\sigma_{2}+\gamma_{2}}{\beta(A / \mu)}\right)$, then the derivative of $V_{1}$ with respect to $t$ along the solution of (7) on the set $\omega$ is given by

$$
\frac{d V_{1}}{d t}=-\rho\left(\mu+\sigma_{1}+\gamma_{1}\right) E(t)+(1-\rho \varepsilon) E(t-\tau)+\left\{\rho \beta \frac{A}{\mu}-\left(\mu+\alpha+c+\sigma_{2}+\gamma_{2}\right)\right\} I(t) .
$$

Thus,

$$
\frac{d V_{1}}{d t} \leq(1-\rho \varepsilon) E(t-\tau)+\left\{\rho \beta \frac{A}{\mu}-\left(\mu+\alpha+c+\sigma_{2}+\gamma_{2}\right)\right\} I(t) .
$$

As $\rho \in\left(\frac{1}{\varepsilon}, \frac{\mu+\alpha+c+\sigma_{2}+\gamma_{2}}{\beta(A / \mu)}\right)$, we have $1-\rho \varepsilon<0$, and $\rho \beta \frac{A}{\mu}-\left(\mu+\alpha+c+\sigma_{2}+\gamma_{2}\right)<0$. Therefore we consider a positive number $\sigma$, such that, $1-\rho \varepsilon<\sigma \rho\left(R_{0}-1\right)$ and $1-\rho \varepsilon<0$, and $\rho \beta \frac{A}{\mu}-$ $\left(\mu+\alpha+c+\sigma_{2}+\gamma_{2}\right)<\sigma\left(R_{0}-1\right)$. Thus from (10) we can write

$$
V_{1}(t) \leq V_{1}(0) \exp \left[\sigma\left(R_{0}-1\right) t\right]
$$


where $V_{1}(0)=\rho E(0)+I(0)$, therefore for $R_{0}<1$ we have

$$
\lim _{t \rightarrow \infty} V_{1}(t)=0, \quad \text { i.e., } \quad \lim _{t \rightarrow \infty} E(t)=0=\lim _{t \rightarrow \infty} I(t) .
$$

This shows that the disease will extinct if $R_{0}<1$.

In order to discuss the persistence of the disease, we first introduce some definitions; then we follow the steps in [41].

Assume that $X$ is a locally compact metric space with metric $d$, and let $F$ be a closed subset of $X$ with boundary $\delta F$ and interior int $F$. Let $\pi$ be a semidynamical system defined on $F$. We say that $\pi$ is persistent if, for all $u \in \operatorname{int} F, \lim _{t \rightarrow+\infty} \inf d(\pi(u, t), \delta F)>0$, and we say that $\pi$ is uniformly persistent if there is $\xi>0$ such that, for all $u \in \operatorname{int} F$, $\lim _{t \rightarrow+\infty} \inf d(\pi(u, t), \delta F)>\xi$.

In [41], Fonda gives a result about persistence in terms of repellers. A subset $\Sigma$ of $F$ is said to be a uniform repeller if there is an $\eta>0$ such that, for each $u \in F \backslash \Sigma$, $\lim _{t \rightarrow+\infty} \inf d(\pi(u, t), \Sigma)>\eta$. A semiflow on a closed subset $F$ of a locally compact metric space is uniformly persistent if the boundary of $F$ is repelling in a suitable strong sense.

Lemma 1 Let $\Sigma$ be a compact subset of $X$ such that $X \backslash \Sigma$ is positively invariant. A necessary and sufficient condition for $\Sigma$ to be a uniform repeller is that there exist a neighborhood $\mathbf{U}$ of $\Sigma$ and a continuous function $P: X \rightarrow R_{+}$satisfying

(1) $P(u)=0$ if and only if $u \in \Sigma$,

(2) for all $u \in \mathbf{U} \backslash \Sigma$ there is a $T_{u}>0$ such that $P\left(\pi\left(u, T_{u}\right)\right)>P(u)$.

For any $u_{0}=\left(S_{0}, E_{0}, I_{0}\right) \in \omega$, there is a unique solution $\pi\left(u_{0}, t\right)=(S, E, I)\left(t ; u_{0}\right)$ of system (7), which is defined in $R_{+}$and satisfies $\pi\left(u_{0}, 0\right)=\left(S_{0}, E_{0}, I_{0}\right)$. Since $\omega$ is a positively invariant set of system (7), then $\pi\left(u_{0}, t\right) \in \omega$ for $t \in R_{+}$and it is a semidynamical system in $\omega$. Here, we will prove that, when $R_{0}>1, \Sigma=\{(S, E, I) \in \Sigma: I=0\}$ is a uniform repeller, which implies that the semidynamical system $\pi$ is uniformly persistent. Obviously, $I(t)>0$ for $t>0$ if $I(0)>0$, then $\omega \backslash \Sigma$ is invariant to (7). Again the set $\Sigma$ is a compact subset of $\omega$.

Let $P: \omega \rightarrow R_{+}$be defined by $P(S, E, I)=I$, and let $\mathbf{U}=\left\{(S, E, I) \in \omega: P(S, E, I)<\zeta_{1}\right\}$, where $\zeta_{1}>0$ is small enough so that

$$
\begin{aligned}
& \left\{A-\frac{\mu\left(\mu+\sigma_{1}+\gamma_{1}+\varepsilon\right)\left(\mu+\alpha+c+\sigma_{2}+\gamma_{2}\right)}{\beta \varepsilon}\right\} \\
& \quad-\left\{\frac{\left(\mu+\sigma_{1}+\gamma_{1}+\varepsilon\right)\left(\mu+\alpha+c+\sigma_{2}+\gamma_{2}\right)}{\varepsilon}-c\right\} \zeta_{1}>0 .
\end{aligned}
$$

Since $R_{0}>1$, the positive number $\zeta_{1}$ is sufficiently small to satisfy the inequality (12).

Assume that there is $\bar{u} \in \mathbf{U}(\bar{u}=(\bar{S}, \bar{E}, \bar{I}))$ such that for each $t>0$ we have $P(\pi(\bar{u}, t))<$ $P(\bar{u})<\zeta_{1}$, which implies that $I(t, \bar{u})<\zeta_{1}$ for $t>0$. From the first equation of (7) we have

$$
\frac{d S}{d t} \geq A+c \zeta_{1}-\left(\mu+\beta \zeta_{1}\right) S
$$

then

$$
\lim _{t \rightarrow \infty} \inf S(t, \bar{u}) \geq \frac{A+c \zeta_{1}}{\mu+\beta \zeta_{1}} .
$$


For sufficiently large number of $T>0$ we have $S(t, \bar{u})>\frac{A+c \zeta_{1}}{\mu+\beta \zeta_{1}}$ for $t \geq T$.

Now we define another function $V_{2}(t)=\left(1-\zeta_{2}\right) E(t)+\frac{\mu+\sigma_{1}+\gamma_{1}+\varepsilon}{\beta} I(t)$, where $\zeta_{2}\left(0<\zeta_{2}<1\right)$ is a sufficiently small constant so that

$$
\begin{gathered}
\left\{A-\frac{\mu\left(1-\zeta_{2}\right)\left(\mu+\sigma_{1}+\gamma_{1}+\varepsilon\right)\left(\mu+\alpha+c+\sigma_{2}+\gamma_{2}\right)}{\beta \varepsilon}\right\} \\
>\left\{\frac{\left(\mu+\sigma_{1}+\gamma_{1}+\varepsilon\right)\left(\mu+\alpha+c+\sigma_{2}+\gamma_{2}\right)}{\varepsilon}-c\right\} \zeta_{1} .
\end{gathered}
$$

Now we differentiate $V_{2}(t)$ along with $\pi(\bar{u}, t)$ as follows:

$$
\begin{aligned}
\frac{d V_{2}}{d t} \geq & \frac{\zeta_{2}\left(\mu+\sigma_{1}+\gamma_{1}\right)}{\beta} E(t)+\left\{A-\frac{\mu\left(1-\zeta_{2}\right)\left(\mu+\sigma_{1}+\gamma_{1}+\varepsilon\right)\left(\mu+\alpha+c+\sigma_{2}+\gamma_{2}\right)}{\beta \varepsilon}\right. \\
& \left.-\left(\frac{\left(\mu+\sigma_{1}+\gamma_{1}+\varepsilon\right)\left(\mu+\alpha+c+\sigma_{2}+\gamma_{2}\right)}{\varepsilon}-c\right) \zeta_{1}\right\} I(t), \\
\frac{d V_{2}}{d t}> & \kappa V_{2},
\end{aligned}
$$

where

$$
\begin{aligned}
\kappa= & \min \left\{\frac{\zeta_{2}\left(\mu+\sigma_{1}+\gamma_{1}\right)}{\beta\left(1-\zeta_{2}\right)},\right. \\
& \frac{\beta}{\mu+\sigma_{1}+\gamma_{1}}\left[A-\frac{\mu\left(1-\zeta_{2}\right)\left(\mu+\sigma_{1}+\gamma_{1}+\varepsilon\right)\left(\mu+\alpha+c+\sigma_{2}+\gamma_{2}\right)}{\beta \varepsilon}\right. \\
& \left.\left.-\left(\frac{\left(\mu+\sigma_{1}+\gamma_{1}+\varepsilon\right)\left(\mu+\alpha+c+\sigma_{2}+\gamma_{2}\right)}{\varepsilon}-c\right) \zeta_{1}\right]\right\}>0 .
\end{aligned}
$$

Thus we see that

$$
\lim _{t \rightarrow \infty} V_{2}(t)=+\infty
$$

Therefore, this proof shows that, for each $u \in \omega \backslash \Sigma$ with $u$ belonging to a suitable small neighborhood of $\Sigma$, there is some $T_{u}$ such that $P\left(\pi\left(u, T_{u}\right)\right)>P(u)$. Therefore, it follows from Lemma 1 that $\Sigma=\{(S, E, I) \in \Sigma: I=0\}$ is a uniform repeller when $R_{0}>1$, i.e., the infection is uniformly persistent. So we conclude that system (7) will be persistent for $R_{0}>1$ and infection will be extinct when $R_{0}<1$.

\section{Stability analysis}

Based on the analysis in [29], we know that if $R_{0}>1$, then system (2) has a unique endemic equilibrium $P^{*}\left(S^{*}, E^{*}, I^{*}, Q^{*}\right)$, where

$$
\begin{aligned}
& S^{*}=\frac{A}{\mu R_{0}}, I^{*}=\frac{\varepsilon E^{*}}{\mu+\alpha+c+\sigma_{2}+\gamma_{2}}, \\
& E^{*}=\frac{A\left(R_{0}-1\right)\left(\mu+\alpha+c+\sigma_{2}+\gamma_{2}\right)}{R_{0}\left[\left(\mu+\sigma_{1}+\gamma_{1}\right)\left(\mu+\alpha+c+\sigma_{2}+\gamma_{2}\right)+\varepsilon\left(\mu+\alpha+\sigma_{2}+\gamma_{2}\right)\right]}, \\
& Q^{*}=\frac{\left(\mu+\alpha+c+\sigma_{2}+\gamma_{2}\right) \sigma_{1}+\varepsilon \sigma_{2}}{\left(\mu+\alpha+\gamma_{3}\right)\left(\mu+\alpha+c+\sigma_{2}+\gamma_{2}\right)} E^{*},
\end{aligned}
$$




$$
R_{0}=\frac{A \beta \varepsilon}{\mu\left(\mu+\varepsilon+\sigma_{1}+\gamma_{1}\right)\left(\mu+\alpha+c+\sigma_{2}+\gamma_{2}\right)} .
$$

In this section the linear stability of system (2) is discussed by constructing a suitable Lyapunov functional given in (20). For this purpose, let $u_{1}(t)=S(t)-S^{*}, u_{2}(t)=E(t)-E^{*}$, $u_{3}(t)=I(t)-I^{*}, u_{4}(t)=Q(t)-Q^{*}$ then system (2) transforms into

$$
\left\{\begin{array}{l}
\frac{d u_{1}(t)}{d t}=\left(-\beta I^{*}-\mu\right) u_{1}+\left(c-\beta S^{*}\right) u_{3} \\
\frac{d B_{1}(t)}{d t}=\beta I^{*} u_{1}-\left(\mu+\sigma_{1}+\gamma_{1}+\varepsilon\right) u_{2}+\beta S^{*} u_{3} \\
\frac{d B_{2}(t)}{d t}=\varepsilon u_{2}-\left(\mu+\alpha+c+\sigma_{2}+\gamma_{2}\right) u_{3} \\
\frac{d u_{4}}{d t}=\sigma_{1} u_{2}+\sigma_{2} u_{3}-\left(\mu+\alpha+\gamma_{3}\right) u_{4}
\end{array}\right.
$$

where $B_{1}(t)=u_{2}-\varepsilon \int_{t-\tau}^{t} u_{2}(s) d s$ and $B_{2}(t)=u_{3}+\varepsilon \int_{t-\tau}^{t} u_{2}(s) d s$.

Now following the steps in $[42,43]$, we shall check the stability of the system by assuming a suitable Lyapunov function $w(v)(t)$ as follows:

$$
\begin{aligned}
w(u)(t)= & k_{1} w_{1}(u)(t)+k_{2} w_{2}(u)(t)+k_{3} w_{3}(u)(t)+k_{4} w_{4}(u)(t)+k_{5} w_{5}(u)(t) \\
& +k_{6} w_{6}(u)(t)+k_{7} w_{7}(u)(t)+k_{8} w_{8}(u)(t)+k_{9} w_{9}(u)(t)+k_{10} w_{10}(u)(t),
\end{aligned}
$$

where $k_{1}, k_{2}, k_{3}, k_{4}, k_{5}, k_{6}, k_{7}, k_{8}, k_{9}, k_{10}$ are given in the Appendix and

$$
\begin{aligned}
w_{1}(u)(t)= & u_{1}^{2}(t), \\
w_{2}(u)(t)= & B_{1}^{2}(t)+\varepsilon\left(\mu+\sigma_{1}+\gamma_{1}+\varepsilon-\beta I^{*}-\beta S^{*}\right) \int_{t-\tau}^{t} \int_{s}^{t} u_{2}^{2}(l) d l d s, \\
w_{3}(u)(t)= & B_{2}^{2}(t)+\left\{\varepsilon^{2}-\varepsilon\left(\mu+\alpha+c+\sigma_{2}+\gamma_{2}\right)\right\} \int_{t-\tau}^{t} \int_{s}^{t} u_{2}^{2}(l) d l d s, \\
w_{4}(u)(t)= & u_{4}^{2}(t), \\
w_{5}(u)(t)= & u_{1}(t) B_{1}(t)+\frac{\beta I^{*} \varepsilon+\mu \varepsilon-c \varepsilon+\beta S^{*} \varepsilon}{2} \int_{t-\tau}^{t} \int_{s}^{t} u_{2}^{2}(l) d l d s, \\
w_{6}(u)(t)= & u_{1}(t) B_{2}(t)+\frac{c \varepsilon-\beta S^{*} \varepsilon-\beta I^{*} \varepsilon-\mu \varepsilon}{2} \int_{t-\tau}^{t} \int_{s}^{t} u_{2}^{2}(l) d l d s, \\
w_{7}(u)(t)= & u_{1}(t) u_{4}(t), \\
w_{8}(u)(t)= & B_{1}(t) B_{2}(t)+\frac{\beta I^{*} \varepsilon-\sigma_{1} \varepsilon-\gamma_{1} \varepsilon-2 \varepsilon^{2}+\beta S^{*} \varepsilon+\alpha \varepsilon+c \varepsilon+\sigma_{2} \varepsilon+\gamma_{2} \varepsilon}{2} \\
& \times \int_{t-\tau}^{t} \int_{s}^{t} u_{2}^{2}(l) d l d s, \\
w_{9}(u)(t)= & B_{1}(t) u_{4}(t)+\frac{\mu \varepsilon+\alpha \varepsilon+\gamma_{3} \varepsilon-\sigma_{1} \varepsilon-\sigma_{2} \varepsilon}{2} \int_{t-\tau}^{t} \int_{s}^{t} u_{2}^{2}(l) d l d s, \\
w_{10}(u)(t)= & B_{2}(t) u_{4}+\frac{\sigma_{1} \varepsilon+\sigma_{2} \varepsilon-\mu \varepsilon-\alpha \varepsilon-\gamma_{3} \varepsilon}{2} \int_{t-\tau}^{t} \int_{s}^{t} u_{2}^{2}(l) d l d s .
\end{aligned}
$$

All the parameters are assumed to be positive and chosen in such a way that $k_{1}>0$, $k_{2}>0, k_{3}>0, k_{4}>0, k_{5}>0, k_{6}>0, k_{7}>0, k_{8}>0, k_{9}>0, k_{10}>0$ and $w(u)(t)>0$. Taking the 

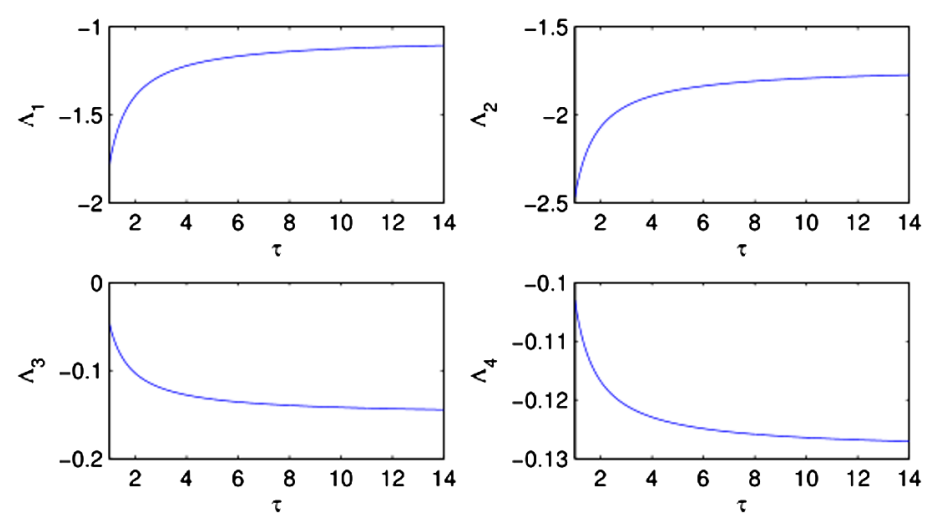

Figure $1 \tau$ versus $\Lambda_{1}, \Lambda_{2}, \Lambda_{3}, \Lambda_{4}$ have been plotted, where all the $\Lambda_{1}, \Lambda_{2}, \Lambda_{3}, \Lambda_{4}$ are negative for $\tau \leq \tau_{0}=13.9858$
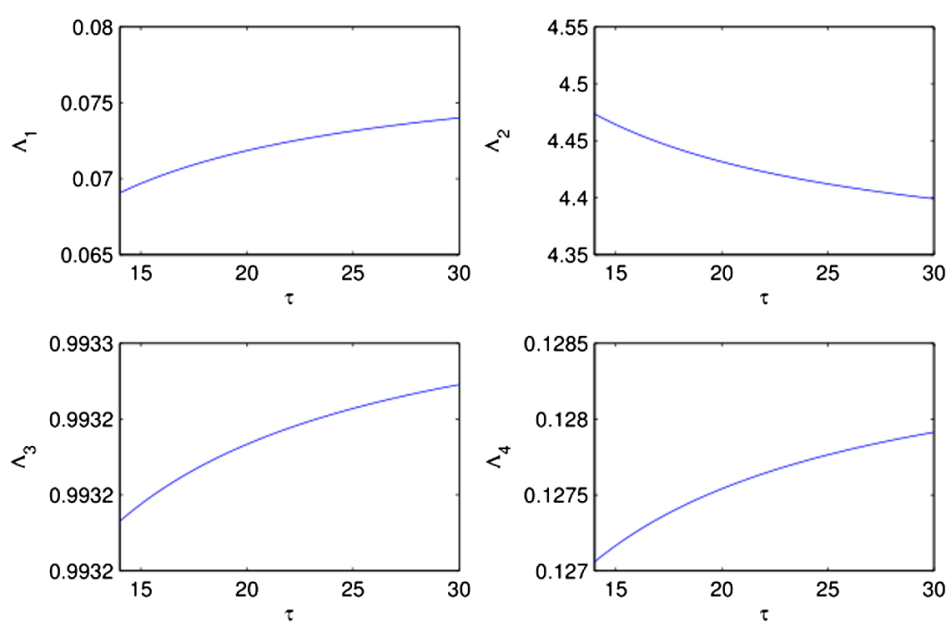

Figure $2 \tau$ versus $\Lambda_{1}, \Lambda_{2}, \Lambda_{3}, \Lambda_{4}$ have been plotted, where all the $\Lambda_{1}, \Lambda_{2}, \Lambda_{3}, \Lambda_{4}$ are positive and $\tau \geq \tau_{0}=13.9858$

derivative of (20), and using (19) we get

$$
\frac{d}{d t} w(u)(t) \leq \Lambda_{1} u_{1}^{2}+\Lambda_{2} u_{2}^{2}+\Lambda_{3} u_{3}^{2}+\Lambda_{4} u_{4}^{2}
$$

where the expressions for $\Lambda_{1}, \Lambda_{2}, \Lambda_{3}, \Lambda_{4}$ are given in the Appendix.

Theorem 1 If the value of the delay $\tau$ satisfy the conditions $\Lambda_{1}<0, \Lambda_{2}<0, \Lambda_{3}<0, \Lambda_{4}<0$ then the interior equilibrium point $P^{*}\left(S^{*}, E^{*}, I^{*}, Q^{*}\right)$ of (2) is locally asymptotically stable (Fig. 1). Otherwise if any one of the $\Lambda_{i}$ become positive then the system will be unstable (Fig. 2)

Proof Let $\Lambda=\max \left\{\Lambda_{1}, \Lambda_{2}, \Lambda_{3}, \Lambda_{4}\right\}$. Then, for $t>T$, from (21) we get

$$
w(u)(t)+\Lambda \int_{T}^{t}\left(u_{1}^{2}(s)+u_{2}^{2}(s)+u_{3}^{2}(s)+u_{4}^{2}(s)\right) d s \leq w(u)(T),
$$


for $t \geq T$, implies $u_{1}^{2}+u_{2}^{2}+u_{3}^{2}+u_{4}^{2} \in L_{1}[T, \infty]$. It is easy to conclude from (19) and the boundedness of $u(t)$ that $u_{1}^{2}(t)+u_{2}^{2}(t)+u_{3}^{2}(t)+u_{4}^{2}(t)$ is uniformly continuous. Using Barbalat's lemma [44], we can say that

$$
\lim _{t \rightarrow \infty}\left\{u_{1}^{2}+u_{2}^{2}+u_{3}^{2}+u_{4}^{2}\right\}=0
$$

So the internal solution of (19) and the solutions of (2) are asymptotically stable, i.e., the positive equilibrium $P^{*}$ of (2) is locally asymptotically stable. Hence, this completes the proof.

Remark As $\Lambda_{1}, \Lambda_{2}, \Lambda_{3}, \Lambda_{4}$ depends on the delay $\tau$ and the local stability condition for $P^{*}$ of system (2) is preserved for small $\tau$ satisfying $\Lambda_{1}<0, \Lambda_{2}<0, \Lambda_{3}<0, \Lambda_{4}<0$. For a set of parameters, $\Lambda_{1}, \Lambda_{2}, \Lambda_{3}, \Lambda_{4}$ have been plotted in Fig. 1, it shows that all the values of $\Lambda$ are negative within an interval of $\tau$, which implies the stability of the system. But, for increased values of the latent delay $\tau$, all the values of $\Lambda$ are positive (see Fig. 2), which shows that the system is unstable.

\section{Linear stability and Hopf-bifurcation analysis}

Here we shall discuss the condition for linear stability and then taking $\tau$ as bifurcation parameter the condition for Hopf bifurcation is discussed. The characteristic equation of system (2) is

$$
\lambda^{4}+m_{3} \lambda^{3}+m_{2} \lambda^{2}+m_{1} \lambda+m_{0}+\left(n_{3} \lambda^{3}+n_{2} \lambda^{2}+n_{1} \lambda+n_{0}\right) e^{-\lambda \tau}=0,
$$

where

$$
\begin{aligned}
& m_{0}=a_{1} a_{4} a_{6} a_{9}, \\
& m_{1}=-\left[a_{1} a_{4} a_{6}+a_{9}\left(a_{1} a_{4}+a_{1} a_{6}+a_{4} a_{6}\right)\right], \\
& m_{2}=a_{1} a_{4}+a_{1} a_{6}+a_{4} a_{6}+a_{9}\left(a_{1}+a_{4}+a_{6}\right), \\
& m_{3}=-\left(a_{1}+a_{4}+a_{6}+a_{9}\right), \\
& n_{0}=a_{1} a_{9}\left(a_{6} b_{1}-a_{5} b_{2}\right)+a_{2} a_{3} a_{9} b_{2}, \\
& n_{1}=a_{5} b_{2}\left(a_{1}+a_{9}\right)-a_{2} a_{3} b_{2}-b_{1}\left(a_{1} a_{6}+a_{1} a_{9}+a_{6} a_{9}\right), \\
& n_{2}=b_{1}\left(a_{1}+a_{6}+a_{9}\right)-a_{5} b_{2}, \quad n_{3}=-b_{1},
\end{aligned}
$$

and

$$
\begin{aligned}
& a_{1}=-\left(\beta I^{*}+\mu\right), \quad a_{2}=c-\beta S^{*}, \quad a_{3}=\beta I^{*}, \\
& a_{4}=-\left(\mu+\sigma_{1}+\gamma_{1}\right), \quad a_{5}=\beta S^{*}, \\
& a_{6}=-\left(\mu+\alpha+c+\sigma_{2}+\gamma_{2}\right), \\
& a_{7}=\sigma_{1}, \quad a_{8}=\sigma_{2}, a_{9}=-\left(\mu+\alpha+\gamma_{3}\right), \quad b_{1}=-\varepsilon, \quad b_{2}=\varepsilon .
\end{aligned}
$$

Theorem 2 For system (2), if $R_{0}>1$ and the conditions $\left(\mathrm{H}_{1}\right)-\left(\mathrm{H}_{2}\right)$ hold, then the endemic equilibrium $P^{*}\left(S^{*}, E^{*}, I^{*}, Q^{*}\right)$ is locally asymptotically stable when $\tau \in\left[0, \tau_{0}\right)$; system (2) 
undergoes a Hopf bifurcation at the endemic equilibrium $P^{*}\left(S^{*}, E^{*}, I^{*}, Q^{*}\right)$ when $\tau=\tau_{0}$ and a family of periodic solutions bifurcate from the endemic equilibrium $P^{*}\left(S^{*}, E^{*}, I^{*}, Q^{*}\right)$. The conditions $\left(\mathrm{H}_{1}\right)$ and $\left(\mathrm{H}_{2}\right)$ are described in the following.

Proof The proof proceeds by using some lemmas.

Lemma 2 ([29]) When $R_{0}>1$, the unique endemic equilibrium $P^{*}\left(S^{*}, E^{*}, I^{*}, R^{*}\right)$ is locally asymptotically stable when $\tau=0$ for system (2).

For $\tau>0$, let $\lambda=i \omega(\omega>0)$ be the root of Eq. (23), then

$$
\left\{\begin{array}{l}
\left(n_{1} \omega-n_{3} \omega^{3}\right) \sin \tau \omega+\left(n_{0}-n_{2} \omega^{2}\right) \cos \tau \omega=m_{2} \omega^{2}-\omega^{4}-m_{0}, \\
\left(n_{1} \omega-n_{3} \omega^{3}\right) \cos \tau \omega-\left(n_{0}-n_{2} \omega^{2}\right) \sin \tau \omega=m_{3} \omega^{3}-m_{1} \omega
\end{array}\right.
$$

which leads to

$$
\omega^{8}+l_{3} \omega^{6}+l_{2} \omega^{4}+l_{1} \omega^{2}+l_{0}=0
$$

where

$$
\begin{aligned}
& l_{0}=m_{0}^{2}-n_{0}^{2}, \\
& l_{1}=m_{1}^{2}-2 m_{0} m_{2}+2 n_{0} n_{2}-n_{1}^{2}, \\
& l_{2}=m_{2}^{2}+2 m_{0}-2 m_{1} m_{3}-n_{2}^{2}+2 n_{1} n_{3}, \\
& l_{3}=m_{3}^{2}-n_{3}^{2}-2 m_{2} .
\end{aligned}
$$

Let $\omega^{2}=v$, then Eq. (25) becomes

$$
v^{4}+l_{3} v^{3}+l_{2} v^{2}+l_{1} v+l_{0}=0
$$

Define

$$
f(v)=v^{4}+l_{3} v^{3}+l_{2} v^{2}+l_{1} v+l_{0}
$$

Thus,

$$
f^{\prime}(v)=4 v^{3}+3 l_{3} v^{2}+2 l_{2} v+l_{1}
$$

Set

$$
4 v^{3}+3 l_{3} v^{2}+2 l_{2} v+l_{1}=0 .
$$

Let $y=v+\frac{3 l_{3}}{4}$. Then Eq. (29) becomes

$$
y^{3}+r_{1} y+s_{1}=0
$$


where

$$
r_{1}=\frac{l_{2}}{2}-\frac{3}{16} l_{3}^{2}, \quad s_{1}=\frac{l_{3}^{3}}{32}-\frac{l_{2} l_{3}}{8}+l_{1} .
$$

Denote

$$
\begin{aligned}
& D=\left(\frac{s_{1}}{2}\right)^{2}+\left(\frac{r_{1}}{3}\right)^{3}, \quad \sigma=\frac{-1+\sqrt{3} i}{2}, \\
& y_{1}=\sqrt[3]{-\frac{s_{1}}{2}+\sqrt{D}}+\sqrt[3]{-\frac{s_{1}}{2}-\sqrt{D}}, \\
& y_{2}=\sqrt[3]{-\frac{s_{1}}{2}+\sqrt{D}} \sigma+\sqrt[3]{-\frac{s_{1}}{2}-\sqrt{D}} \sigma^{2}, \\
& y_{3}=\sqrt[3]{-\frac{s_{1}}{2}+\sqrt{D}} \sigma^{2}+\sqrt[3]{-\frac{s_{1}}{2}-\sqrt{D}} \sigma \\
& v_{i}=y_{i}-\frac{3 l_{3}}{4}, \quad i=1,2,3 .
\end{aligned}
$$

Based on the discussion of the distribution of roots of Eq. (26) in Lemma 2.1 and Lemma 2.2 in [45], we have the following results.

Lemma 3 For Eq. (26), we have

$\left(\mathrm{H}_{1}\right)$ If $l_{0}<0, E q$. (26) has at least one positive root;

$\left(\mathrm{H}_{2}\right)$ If $l_{0} \geq 0$ and $D \geq 0$, Eq. (26) has positive roots if and only if $v_{1}>0$ and $f\left(v_{1}\right)<0$;

$\left(\mathrm{H}_{3}\right)$ If $l_{0} \geq 0$ and $D<0, E q$. (26) has positive roots if and only if there exists at least one $v_{*} \in\left\{v_{1}, v_{2}, v_{3}\right\}$ such that $v_{*}>0$ and $f\left(v_{*}\right) \leq 0$.

In what follows, we assume $\left(\mathrm{H}_{1}\right)$ : the coefficients in $f(v)$ satisfy one of the following conditions in (a) - (c).

(a) $l_{0}<0$;

(b) $l_{0} \geq 0, D \geq 0, v_{1}>0$ and $f\left(v_{1}\right)<0$;

(c) $l_{0} \geq 0, D<0$, and there exists at least one $v_{*} \in\left\{v_{1}, v_{2}, v_{3}\right\}$ such that $v_{*}>0$ and $f\left(v_{*}\right) \leq 0$.

If the condition $\left(\mathrm{H}_{1}\right)$ holds, then Eq. (26) has positive root $v_{0}$ such that Eq. (23) has a pair of imaginary roots $\pm i \omega_{0}= \pm i \sqrt{v_{0}}$. Further, we have

$$
\tau_{0}=\frac{1}{\omega_{0}} \arccos \left\{\frac{\left(n_{2}-m_{3} n_{3}\right) \omega_{0}^{6}+\left(m_{1} n_{3}+m_{3} n_{1}-m_{2} n_{2}\right) \omega_{0}^{4}+\left(m_{0} n_{2}+m_{2} n_{0}-m_{1} n_{1}\right) \omega_{0}^{2}-m_{0} n_{0}}{\left(n_{1} \omega_{0}-n_{3} \omega_{0}^{3}\right)^{2}+\left(n_{0}-n_{2} \omega_{0}^{2}\right)^{2}}\right\} .
$$

Differentiating both sides of Eq. (23) with respect to $\tau$ yields

$$
\left[\frac{d \lambda}{d \tau}\right]^{-1}=-\frac{4 \lambda^{3}+3 m_{3} \lambda^{2}+2 m_{2} \lambda+m_{1}}{\lambda\left(\lambda^{4}+m_{3} \lambda^{3}+m_{2} \lambda^{2}+m_{1} \lambda+m_{0}\right)}+\frac{3 n_{3} \lambda^{2}+2 n_{2} \lambda+n_{1}}{\lambda\left(n_{3} \lambda^{3}+n_{2} \lambda^{2}+n_{1} \lambda+n_{0}\right)}-\frac{\tau}{\lambda} .
$$

Further, we have

$$
\operatorname{Re}\left[\frac{d \lambda}{d \tau}\right]_{\tau=\tau_{0}}^{-1}=\frac{f^{\prime}\left(v_{* *}\right)}{\left(n_{1} \omega_{0}-n_{3} \omega_{0}^{3}\right)^{2}+\left(n_{0}-n_{2} \omega_{0}^{2}\right)^{2}}
$$

where $f(v)=v^{4}+l_{3} v^{3}+l_{2} v^{2}+l_{1} v+l_{0}$ and $v_{* *}=\omega_{0}^{2}$. 
Clearly, if the condition $\left(\mathrm{H}_{2}\right): f^{\prime}\left(\omega_{0}^{2}\right) \neq 0$ holds, then $\operatorname{Re}[d \lambda / d \tau]_{\tau=\tau_{0}}^{-1} \neq 0$. Therefore, by the Hopf-bifurcation theorem that determines the existence of a Hopf bifurcation for a delayed dynamical system in [44], we can obtain the results described in Theorem 2. The proof is completed.

\section{Direction of the Hopf bifurcation and stability of the periodic solutions}

Let $u_{1}(t)=S(t)-S^{*}, u_{2}(t)=E(t)-E^{*}, u_{3}(t)=I(t)-I^{*}, u_{4}(t)=Q(t)-Q^{*}$, and normalize the delay with $t \rightarrow(t / \tau)$. Let $\tau=\tau_{0}+\varrho(\varrho \in R)$, then $\varrho=0$ is the Hopf-bifurcation value of system (2). And system (2) can be transformed into a functional differential equation in $C=C\left([-1,0], R^{4}\right)$ as follows:

$$
\dot{u}(t)=L_{\varrho}\left(u_{t}\right)+F\left(\varrho, u_{t}\right)
$$

where $u(t)=\left(u_{1}, u_{2}, u_{3}, u_{4}\right)^{T} \in C=C\left([-1,0], R^{4}\right)$ and $L_{\varrho}: C \rightarrow R^{4}$ and $F: R \times C \rightarrow R^{4}$ are given, respectively, by

$$
L_{\varrho} \phi=\left(\tau_{0}+\varrho\right)\left(A_{\max } \phi(0)+B_{\max } \phi(-1)\right)
$$

and

$$
F(\varrho, \phi)=\left(\begin{array}{c}
-\beta \phi_{1}(0) \phi_{3}(0) \\
\beta \phi_{1}(0) \phi_{3}(0) \\
0 \\
0
\end{array}\right)
$$

with

$$
A_{\max }=\left(\begin{array}{cccc}
a_{1} & 0 & a_{2} & 0 \\
a_{3} & a_{4} & a_{5} & 0 \\
0 & 0 & a_{6} & 0 \\
0 & a_{7} & a_{8} & a_{9}
\end{array}\right), \quad B_{\max }=\left(\begin{array}{cccc}
0 & 0 & 0 & 0 \\
0 & b_{1} & 0 & 0 \\
0 & b_{2} & 0 & 0 \\
0 & 0 & 0 & 0
\end{array}\right) .
$$

\section{Theorem 3}

(i) If $\mu_{2}>0\left(\mu_{2}<0\right)$, then the Hopf bifurcation is supercritical (subcritical);

(ii) if $\beta_{2}<0\left(\beta_{2}>0\right.$, then the bifurcation periodic solutions are stable (unstable);

(iii) if $T_{2}>0\left(T_{2}<0\right)$, then the bifurcating periodic solutions increase (decrease).

The expressions of $\mu_{2}, \beta_{2}$ and $T_{2}$ are described in the following.

Proof By the Riesz representation theorem, there exists a function $\eta(\theta, \varrho)$ of bounded variation for $\theta \in[-1,0]$ such that

$$
L_{\mu} \phi=\int_{-1}^{0} d \eta(\theta, \varrho) \phi(\theta), \quad \text { for } \phi \in C
$$

In fact, we choose

$$
\eta(\theta, \varrho)=\left(\tau_{0}+\varrho\right)\left(A_{\max } \delta(\theta)+B_{\max } \delta(\theta+1)\right)
$$

where $\delta(\theta)$ is the Dirac delta function. 
For $\phi \in C\left([-1,0], R^{4}\right)$, define

$$
A(\varrho) \phi= \begin{cases}\frac{d \phi(\theta)}{d \theta}, & -1 \leq \theta<0 \\ \int_{-1}^{0} d \eta(\theta, \varrho) \phi(\theta), & \theta=0\end{cases}
$$

and

$$
R(\varrho) \phi= \begin{cases}0, & -1 \leq \theta<0 \\ F(\varrho, \phi), & \theta=0\end{cases}
$$

Then system (32) is equivalent to

$$
\dot{u}(t)=A(\varrho) u_{t}+R(\varrho) u_{t}
$$

For $\varphi \in C^{1}\left([0,1],\left(R^{4}\right)^{*}\right)$, the adjoint operator $A^{*}$ of $A(0)$ is defined as follows:

$$
A^{*}(\varphi)= \begin{cases}-\frac{d \varphi(s)}{d s}, & 0<s \leq 1 \\ \int_{-1}^{0} d \eta^{T}(s, 0) \varphi(-s), & s=0\end{cases}
$$

Next, we define the bilinear inner form for $A$ and $A^{*}$

$$
\langle\varphi(s), \phi(\theta)\rangle=\bar{\varphi}(0) \phi(0)-\int_{\theta=-1}^{0} \int_{\xi=0}^{\theta} \bar{\varphi}(\xi-\theta) d \eta(\theta) \phi(\xi) d \xi
$$

where $\eta(\theta)=\eta(\theta, 0)$.

Let $q(\theta)=\left(1, q_{2}, q_{3}, q_{4}\right)^{T} e^{i \tau_{0} \omega_{0} \theta}$ be the eigenvector of $A(0)$ corresponding to $+i \tau_{0} \omega_{0}$ and $q^{*}(s)=V\left(1, q_{2}^{*}, q_{3}^{*}, q_{4}^{*}\right)^{T} e^{i \tau_{0} \omega_{0} s}$ be the eigenvector of $A^{*}(0)$ corresponding to $-i \tau_{0} \omega_{0}$, respectively. Based on the definition of $A(0)$ and $A^{*}$, one can obtain

$$
\begin{aligned}
& q_{2}=\frac{a_{3}+a_{5} q_{3}}{i \omega_{0}-a_{4}-b_{1} e^{-i \tau_{0} \omega_{0}}}, \quad q_{3}=\frac{i \omega_{0}-a_{1}}{a_{2}}, \quad q_{4}=\frac{a_{7} q_{2}+a_{8} q_{3}}{\left(i \omega_{0}-a_{9}\right)}, \\
& q_{2}^{*}=-\frac{i \omega_{0}+a_{1}}{a_{3}}, \quad q_{4}^{*}=-\frac{a_{2}+a_{5} q_{2}+\left(a_{6}+i \omega_{0}\right) q_{3}}{a_{8}}, \\
& q_{3}^{*}=\frac{a_{7}\left(a_{2}+a_{5} q_{2}\right)-a_{8}\left(i \omega_{0}+a_{4}+b_{1} e^{i \tau_{0} \omega_{0}}\right) q_{2}}{a_{8} b_{2} e^{i \tau_{0} \omega_{0}}-a_{7}\left(a_{6}+i \omega_{0}\right)} .
\end{aligned}
$$

From Eq. (35), we can obtain

$$
\bar{V}=\left[1+q_{2} q_{2}^{*}+q_{3} \bar{q}_{3}^{*}+q_{4} \bar{q}_{4}^{*}+\tau_{0} e^{-i \tau_{0} \omega_{0}} q_{2}\left(b_{1} \bar{q}_{2}^{*}+b_{2} \bar{q}_{3}^{*}\right)\right]^{-1}
$$

such that $\left\langle q^{*}, q\right\rangle=1$ and $\left\langle q^{*}, \bar{q}\right\rangle=0$.

Next, according to the algorithms in [44] and a similar computation process to that in [46], we can obtain the expressions of $g_{20}, g_{11}, g_{02}$ and $g_{21}$ as follows:

$$
\begin{aligned}
& g_{20}=2 \beta \tau_{0} \bar{V} q_{3}\left(\bar{q}_{3}^{*}-1\right), \\
& g_{11}=\beta \tau_{0} \bar{V}\left(q_{3}+\bar{q}_{3}\right)\left(\bar{q}_{3}^{*}-1\right),
\end{aligned}
$$




$$
\begin{aligned}
g_{02}= & 2 \beta \tau_{0} \bar{V} \bar{q}_{3}\left(\bar{q}_{3}^{*}-1\right) \\
g_{21}= & 2 \beta \tau_{0} \bar{V}\left(\bar{q}_{3}^{*}-1\right)\left(W_{11}^{(1)}(0) q_{2}+\frac{1}{2} W_{20}^{(1)}(0) \bar{q}_{2}\right. \\
& \left.+W_{11}^{(3)}(0)+\frac{1}{2} W_{20}^{(3)}(0)\right)
\end{aligned}
$$

with

$$
\begin{aligned}
& W_{20}(\theta)=\frac{i g_{20} q(0)}{\tau_{0} \omega_{0}} e^{i \tau_{0} \omega_{0} \theta}+\frac{i \bar{g}_{02} \bar{q}(0)}{3 \tau_{0} \omega_{0}} e^{-i \tau_{0} \omega_{0} \theta}+E_{1} e^{2 i \tau_{0} \omega_{0} \theta}, \\
& W_{11}(\theta)=-\frac{i g_{11} q(0)}{\tau_{0} \omega_{0}} e^{i \tau_{0} \omega_{0} \theta}+\frac{i \bar{g}_{11} \bar{q}(0)}{\tau_{0} \omega_{0}} e^{-i \tau_{0} \omega_{0} \theta}+E_{2} .
\end{aligned}
$$

$E_{1}$ and $E_{2}$ can be obtained by the following two equations:

$$
\begin{aligned}
& E_{1}=2\left(\begin{array}{cccc}
2 i \omega_{0}-a_{1} & 0 & -a_{2} & 0 \\
-a_{3} & 2 i \omega_{0}-a_{4}-b_{1} e^{-2 i \tau_{0} \omega_{0}} & -a_{5} & 0 \\
0 & -b_{2} e^{-2 i \tau_{0} \omega_{0}} & 2 i \omega_{0}-a_{6} & 0 \\
0 & a_{7} & -a_{8} & 2 i \omega_{0}-a_{9}
\end{array}\right)^{-1} \times\left(\begin{array}{c}
\beta q_{3} \\
\beta q_{3} \\
0 \\
0
\end{array}\right), \\
& E_{2}=-\left(\begin{array}{cccc}
a_{1} & 0 & a_{2} & 0 \\
a_{3} & a_{4}+b_{1} & a_{5} & 0 \\
0 & b_{2} & a_{6} & 0 \\
0 & a_{7} & a_{8} & a_{9}
\end{array}\right)^{-1} \times\left(\begin{array}{c}
-\beta\left(q_{3}+\bar{q}_{3}\right) \\
\beta\left(q_{3}+\bar{q}_{3}\right) \\
0 \\
0
\end{array}\right) .
\end{aligned}
$$

Then one can obtain

$$
\begin{aligned}
& C_{1}(0)=\frac{i}{2 \tau_{0} \omega_{0}}\left(g_{11} g_{20}-2\left|g_{11}\right|^{2}-\frac{\left|g_{02}\right|^{2}}{3}\right)+\frac{g_{21}}{2}, \\
& \mu_{2}=-\frac{\operatorname{Re}\left\{C_{1}(0)\right\}}{\operatorname{Re}\left\{\lambda^{\prime}\left(\tau_{0}\right)\right\}}, \\
& \beta_{2}=2 \operatorname{Re}\left\{C_{1}(0)\right\} \\
& T_{2}=-\frac{\operatorname{Im}\left\{C_{1}(0)\right\}+\mu_{2} \operatorname{Im}\left\{\lambda^{\prime}\left(\tau_{0}\right)\right\}}{\tau_{0} \omega_{0}} .
\end{aligned}
$$

Thus, we can obtain the results described in Theorem 3. The proof is completed.

\section{Numerical simulation}

In this section we shall perform some numerical scenario as the support of our obtained analytical results by choosing the suitable value of the parameters. For different values of delays we obtain different scenarios with $P^{*}\left(S^{*}, E^{*}, I^{*}, Q^{*}\right)$ as interior equilibrium point. The value of the parameters are taken as follows:

$$
\begin{aligned}
& A=20, \quad \beta=0.5, \quad \mu=c=0.25, \quad \varepsilon=0.25, \\
& \sigma_{1}=\gamma_{1}=0.125, \quad \alpha=0.5, \quad \sigma_{2}=\gamma_{2}=0.25, \quad \gamma_{3}=0.5 .
\end{aligned}
$$


The parameters are chosen in such a way that they satisfy the conditions obtained in the previous sections analytically. We have

$$
\left\{\begin{array}{l}
\frac{d S(t)}{d t}=20-0.5 S(t) I(t)-0.25 S(t)+0.25 I(t), \\
\frac{d E(t)}{d t}=0.5 S(t) I(t)-0.5 E(t)-0.5 E(t-\tau), \\
\frac{d I(t)}{d t}=0.5 E(t-\tau)-1.5 I(t) \\
\frac{d Q(t)}{d t}=0.125 E(t)+0.25 I(t)-1.25 Q(t) .
\end{array}\right.
$$

With this set of parameters we get the basic reproduction number $R_{0}=13.3333>0$ and the unique endemic equilibrium $P^{*}(6,20.1818,6.7273,3.3636)$. Biologically it shows that all the individuals coexist. First, in the absence of latent delay, i.e. $\tau=0$ the dynamics of system (1) has been plotted in Fig. 3 and the dynamics is stable in the absence of delay (Lemma 1). But in Fig. 4 it is seen that in the presence of delay (very small value of $\tau$ ) initially all the individuals are oscillating and after some time again it comes to a stable situation. Thus for a more increased value of the delay the oscillation for the individuals also increases. Hence, the interior equilibrium point $P^{*}\left(S^{*}, E^{*}, I^{*}, Q^{*}\right)$ is seen to be stable for $\tau<\tau_{0}$ (Fig. 5) and at the critical value of delay we get a stable periodic solution where the Hopf bifurcation occurs (Fig. 6). Finally for large value of delay $\tau>\tau_{0}$ the system loses
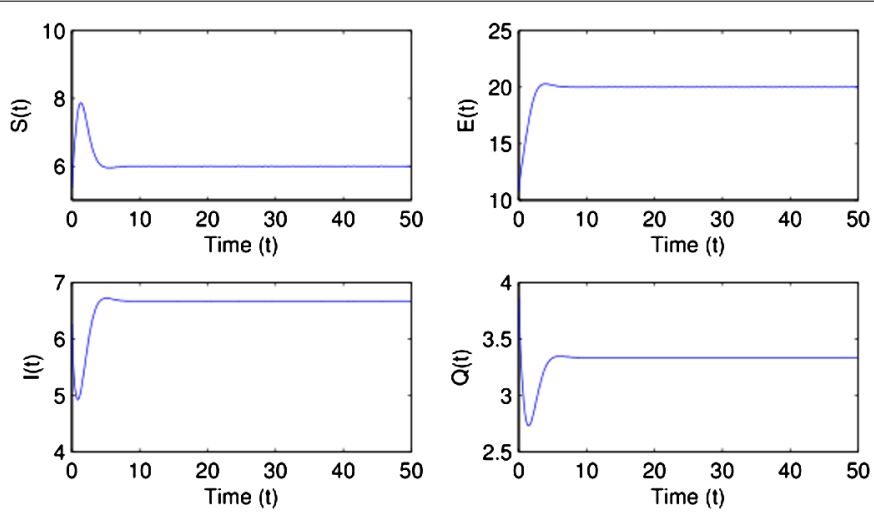

Figure 3 Time series plot for $\tau=0$, which is stable
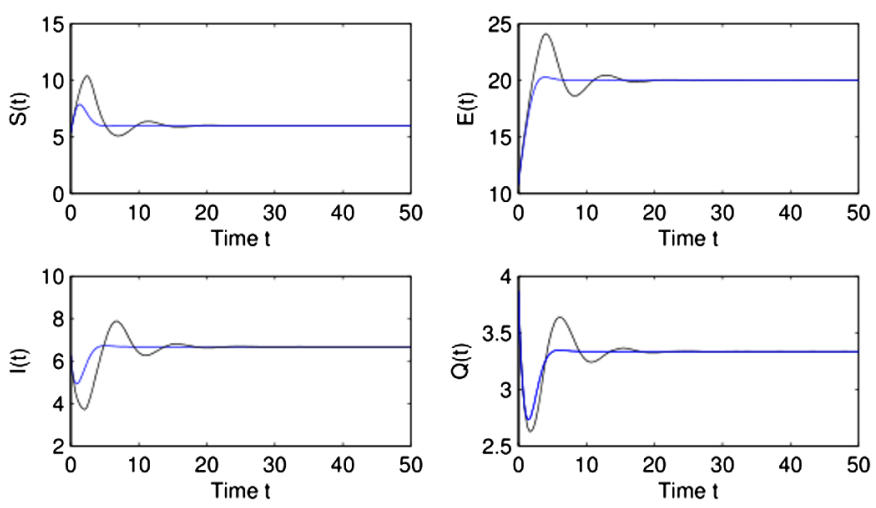

Figure 4 Time series plot for $\tau \neq 0$ but very small $\tau$, which is stable 

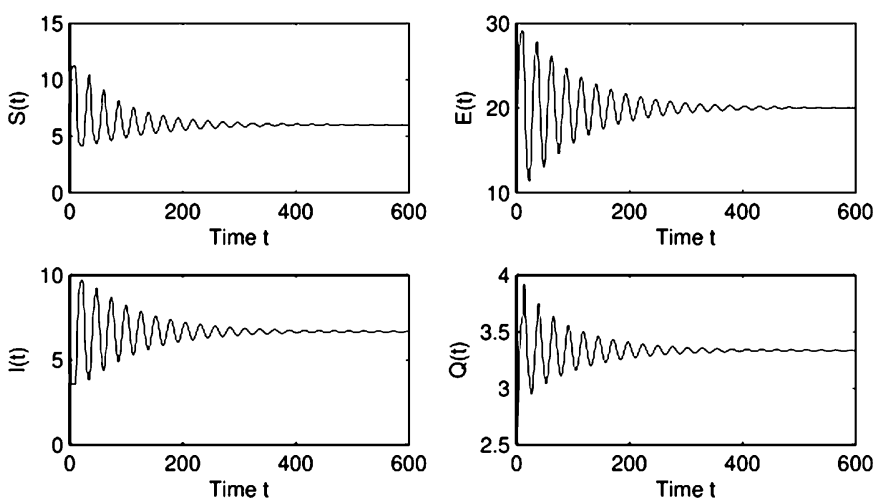

Figure $5 P^{*}$ is locally asymptotically stable when $\tau=10.65<\tau_{0}=13.9858$
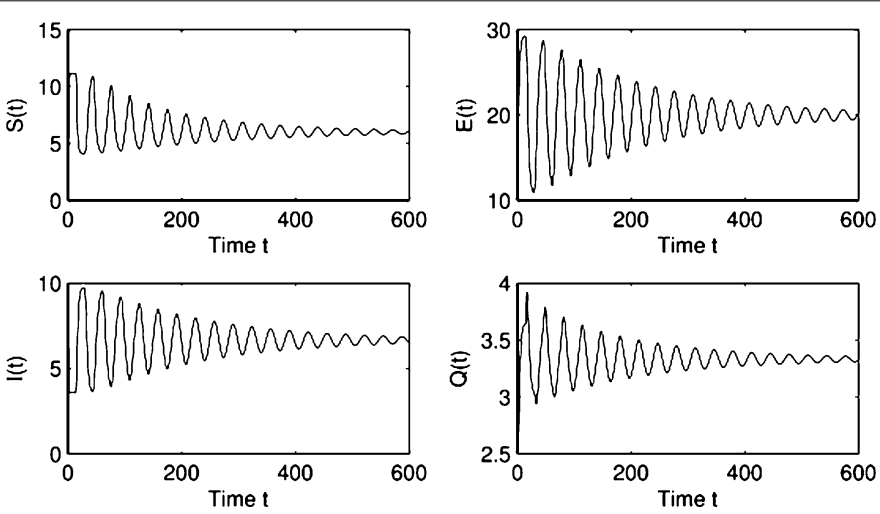

Figure $6 P^{*}$ is stable periodic when $\tau=\tau_{0}=13.9858$
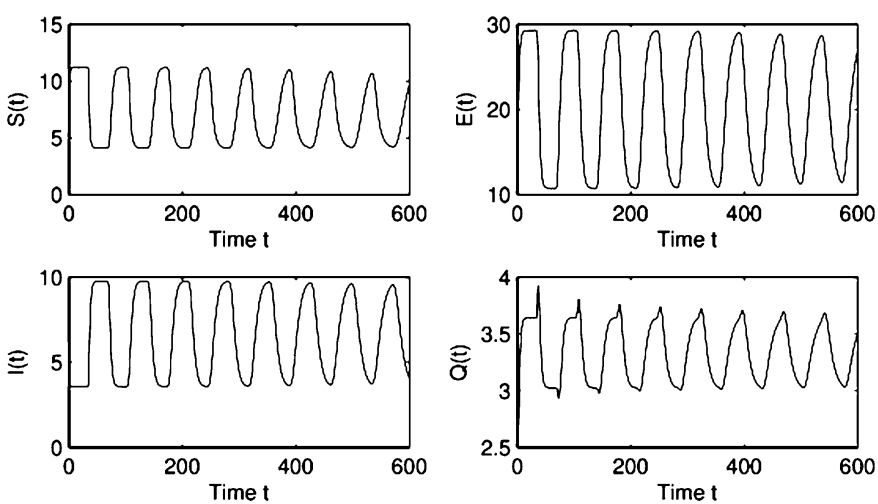

Figure $7 p^{*}$ loses its stability when $\tau=28.85>\tau_{0}=13.9858$

its stability (Fig. 7). This property can be also illustrated by the bifurcation diagram with respect to $\tau$ in Fig. 8 .

\section{Conclusions}

In [29], a non-delayed SEIQ epidemic model has been investigated by the authors. But the disease models are not instantaneous, i.e., infected diseases start by infection and then 


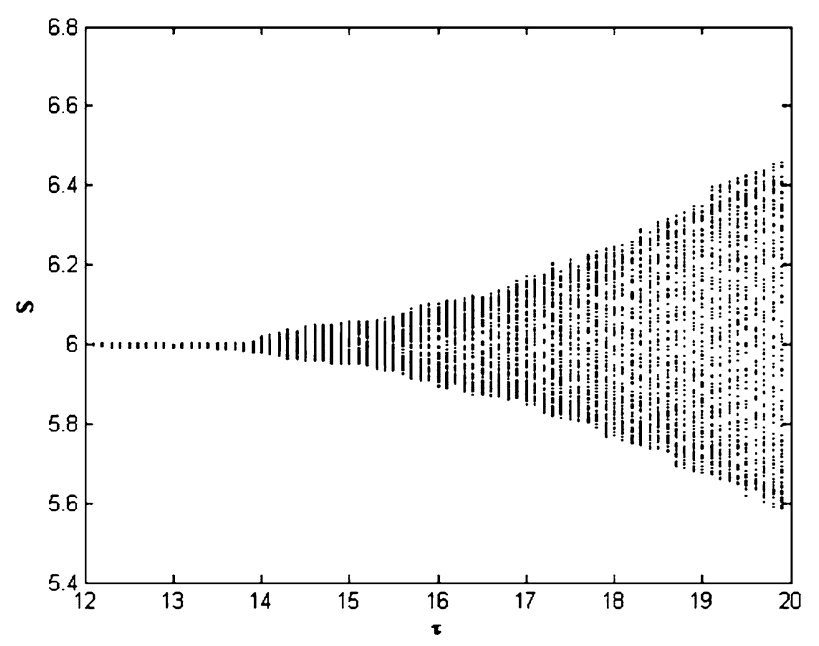

Figure 8 Bifurcation diagram with respect to time delay

some symptoms can be seen. Thus, in the incubation period it takes some time for a response to occur, i.e., delay is arising. Here, in this article we have assumed a delayed SEIQ epidemic model by incorporating the latent delay to the model proposed in [29]. Thus, compared with the model proposed in [29], the model we consider in the present paper is more general. We consider not only the effect of the time delay on the model, but also the boundedness, persistence and the properties of the Hopf bifurcation. The results obtained in the present paper are the complement of the research work in the literature [29].

For this model, a feasible region $\bar{R}$ is obtained with the appropriate choice of the parameters. It can be seen that all the solutions of (2) will remain in or tend to $\bar{R}$, i.e., the feasible region $\bar{R}$ is positive and invariant. If the basic reproduction number $R_{0}>1$, then the model has an endemic equilibrium point which is unique. Also if $R_{0}>1$ the system (2) will be persistent and for $R_{0}<1$, the infection will be extinct, i.e., system (2) becomes disease free. Next, we construct a suitable Lyapunov functional of the form (20) to check the stability of system (2). Using this Lyapunov functional the sufficient conditions for local asymptotic stability are given in Theorem 1 . With the choice of the parameters given in the numerical section and for $\tau<\tau_{0}$ all the $\Lambda$ are negative (Fig. 1), which satisfies the conditions obtained in Theorem 1 . Next, the sufficient conditions for local stability of the endemic equilibrium of the model and the existence of a Hopf bifurcation are obtained by taking the delay as the bifurcating parameter. Also, the critical value of the latent delay is obtained. We can conclude that if the latent delay for system (2) is less than its critical value then the endemic equilibrium for the system gets in a stable situation but if it is greater than the critical value the endemic equilibrium for system (2) will lose its stability. Further, properties of the Hopf bifurcation such as direction and stability are studied by means of the center manifold and normal form theory. At the end, with a set of suitable parameters, some numerical computations are presented to justify our results obtained analytically and to see the effect of the latent delay on the stability of the system.

It should be pointed out that system (2) undergoes a local Hopf bifurcation at the endemic equilibrium $P^{*}\left(S^{*}, E^{*}, I^{*}, Q^{*}\right)$ and a bifurcating periodic solution exists when $\tau$ near 
$\tau_{0}$. In other words, the existence of these periodic solutions remains valid only in a small neighborhood of the critical value $\tau_{0}$. It is interesting to investigate whether these periodic solutions remain when the value of the time delay $\tau$ becomes large enough. We leave the existence of a global Hopf bifurcation of system (2) as our next work in the near future.

\section{Appendix}

We have

$$
\begin{aligned}
& \Lambda_{1}=-2 k_{1}\left(\mu+\beta I^{*}\right)-k_{2} \beta I^{*} \varepsilon \tau+k_{5}\left\{\beta I^{*}+\frac{\beta I^{*} \varepsilon \tau+\mu \varepsilon \tau}{2}\right\} \\
& +k_{6} \frac{-\mu \varepsilon \tau-\beta I^{*} \varepsilon \tau}{2}+k_{8} \frac{\beta I^{*} \varepsilon \tau}{2}+k_{10} \frac{\sigma_{1} \varepsilon \tau}{2}, \\
& \Lambda_{2}=k_{2}\left\{-2 \mu-2 \sigma_{1}-2 \gamma_{1}-2 \varepsilon+\varepsilon \tau\left(2 \mu+2 \sigma_{1}+2 \gamma_{1}+2 \varepsilon-\beta I^{*}-\beta S^{*}\right)\right\} \\
& +k_{3}\left\{2 \tau \varepsilon^{2}-\varepsilon \tau\left(\mu+\alpha+c+\sigma_{2}+\gamma_{2}\right)\right\}+k_{5} \frac{\beta I^{*} \varepsilon \tau+\mu \varepsilon \tau-c \varepsilon \tau+\beta S^{*} \varepsilon \tau}{2} \\
& +k_{6} \frac{-\tau \varepsilon\left(\beta I^{*}+\mu-c+\beta S^{*}\right)}{2} \\
& +k_{8}\left\{\varepsilon+\frac{\tau \varepsilon}{2}\left(-4 \varepsilon-\mu-2 \sigma_{1}-2 \gamma_{1}+\beta I^{*}+\beta S^{*}+\alpha+c+\sigma_{2}+\gamma_{2}\right)\right\} \\
& +k_{9} \frac{2 \sigma_{1}+\tau \varepsilon\left(\mu+\alpha+\gamma_{3}-2 \sigma_{1}-\sigma_{2}\right)}{2}+k_{10} \frac{\sigma_{1} \varepsilon \tau}{2}, \\
& \Lambda_{3}=k_{2}\left(-\beta S^{*} \varepsilon \tau\right)-k_{3}\left\{2\left(\mu+\alpha+c+\sigma_{2}+\gamma_{2}\right)+\tau \varepsilon\left(\mu+\alpha+c+\sigma_{2}+\gamma_{2}\right)\right\} \\
& +k_{5} \frac{\beta S^{*} \varepsilon \tau-c \varepsilon \tau}{2}+k_{6} \frac{2 c-2 \beta S^{*}+c \varepsilon \tau-\beta \varepsilon \tau S^{*}}{2} \\
& +k_{8} \frac{2 \beta S^{*}+\left(\beta S^{*}+\mu+\alpha+c+\sigma_{2}+\gamma_{2}\right) \varepsilon \tau+}{2}+k_{9} \frac{-\varepsilon \sigma_{2} \tau}{2}+k_{10} \frac{2 \sigma_{2}+\sigma_{2} \epsilon \tau}{2}, \\
& \Lambda_{4}=-2 k_{4}\left(\mu+\alpha+\gamma_{3}\right)+k_{9} \frac{\left(\mu+\alpha+\gamma_{3}\right) \tau \varepsilon}{2}+k_{10} \frac{\left(-\mu-\alpha-\gamma_{3}\right) \tau \varepsilon}{2}, \\
& k_{1}=k_{4} \\
& =\left(\gamma_{3} S^{*}+c \tau+\frac{c \gamma_{2} \tau+2 \beta^{2} \tau+2 \mu \alpha}{2}+\frac{\sigma_{1} \sigma_{2}}{2}+2 \beta S^{*}+\frac{c-\beta S^{*}}{2}+\frac{-\beta S^{*} \varepsilon \tau}{2}\right. \\
& \left.+\frac{-\beta S^{*} \varepsilon \tau-c \varepsilon \tau}{2}+\frac{\mu+\alpha+\gamma_{3}}{2}\right) /\left(2 \varepsilon \left(-\gamma_{1}-\beta+\frac{\beta \gamma_{3} \tau}{2}+\frac{\gamma_{1}+\sigma_{2} \tau}{2}+\frac{\beta I^{*}+\mu}{2}\right.\right. \\
& \left.\left.+\frac{\varepsilon \tau\left(-2 \mu-\sigma_{1}-\gamma_{1}-\varepsilon\right)}{2}+\frac{\left(\mu+\alpha+c+\sigma_{2}+\gamma_{2}\right) \tau}{2}+\frac{-2 \mu+\gamma_{1} \gamma_{2} \tau+2 \sigma_{1} \tau}{2}\right)\right) \text {, } \\
& k_{2}=k_{3}=2 k_{8} \\
& =\left(\sigma_{2} I^{*}+\sigma_{2} \gamma_{2} \tau+\frac{\sigma_{1} \gamma_{3} \tau+\beta I^{*}+\beta S^{*} \tau \varepsilon-c \tau \varepsilon}{2}+\frac{-\mu \tau \varepsilon-\beta \varepsilon I^{*} \tau}{2}+\frac{\beta \gamma_{3} \tau}{2}\right. \\
& \left.+\frac{\varepsilon+\alpha+c}{2}+\frac{\sigma_{1}+\alpha+c+2 \gamma_{2}}{2}\right) /\left(2 \varepsilon \left(-\gamma_{1}-\beta+\frac{\beta \gamma_{3} \tau}{2}+\frac{\gamma_{1}+\sigma_{2} \tau}{2}+\frac{\beta I^{*}+\mu}{2}\right.\right. \\
& \left.\left.+\frac{\varepsilon \tau\left(-2 \mu-\sigma_{1}-\gamma_{1}-\varepsilon\right)}{2}+\frac{\left(\mu+\alpha+c+\sigma_{2}+\gamma_{2}\right) \tau}{2}+\frac{-2 \mu+\gamma_{1} \gamma_{2} \tau+2 \sigma_{1} \tau}{2}\right)\right) \text {, } \\
& k_{5}=k_{10}
\end{aligned}
$$




$$
\begin{aligned}
= & 2\left(\mu+\alpha+\gamma_{3}\right)+\frac{\beta S^{*} \tau}{2}+\frac{\beta I^{*} \varepsilon \tau}{2}+2\left(\mu+\beta I^{*}\right)+\frac{\alpha+c+\mu+\gamma_{2} \tau}{2}+2 \sigma_{1}+\varepsilon \tau \\
& +\frac{c-\beta S^{*}-\beta I^{*}-2 \mu}{2}+\frac{\varepsilon \tau}{2}\left(2 \mu+2 \alpha+\gamma_{3}+c+\sigma_{2}+\gamma_{2}\right) /\left(2 \varepsilon \left(-\gamma_{1}-\beta+\frac{\beta \gamma_{3} \tau}{2}\right.\right. \\
& +\frac{\gamma_{1}+\sigma_{2} \tau}{2}+\frac{\beta I^{*}+\mu}{2}+\frac{\varepsilon \tau\left(-2 \mu-\sigma_{1}-\gamma_{1}-\varepsilon\right)}{2} \\
& \left.\left.+\frac{\left(\mu+\alpha+c+\sigma_{2}+\gamma_{2}\right) \tau}{2}+\frac{-2 \mu+\gamma_{1} \gamma_{2} \tau+2 \sigma_{1} \tau}{2}\right)\right), \\
k_{6}= & \left.k_{7}=k_{9}\right) \\
= & \left(-2 \mu-2 \sigma_{1}-2 \gamma_{1}-2 \varepsilon+\tau \varepsilon\left(2 \mu+2 \sigma_{1}+2 \gamma_{1}+2 \varepsilon-\beta I^{*}-\beta S^{*}\right)\right) /\left(2 \varepsilon \left(-\gamma_{1}\right.\right. \\
& -\beta+\frac{\beta \gamma_{3} \tau}{2}+\frac{\gamma_{1}+\sigma_{2} \tau}{2}+\frac{\beta I^{*}+\mu}{2}+\frac{\varepsilon \tau\left(-2 \mu-\sigma_{1}-\gamma_{1}-\varepsilon\right)}{2} \\
& \left.\left.+\frac{\left(\mu+\alpha+c+\sigma_{2}+\gamma_{2}\right) \tau}{2}+\frac{-2 \mu+\gamma_{1} \gamma_{2} \tau+2 \sigma_{1} \tau}{2}\right)\right) .
\end{aligned}
$$

\section{Acknowledgements}

The authors express their gratitude to the referees for their helpful suggestions, which improved the final version of this paper. The first and the second author are thankful to the third author, for her careful guidance, without which this research would not have been possible. The second author is thankful to DST, New Delhi, India, for its financial support under INSPIRE fellowship, without which this research would not have been possible.

\section{Funding}

This work was supported by Project of Support Program for Excellent Youth Talent in Colleges and Universities of Anhui Province (No. gxyqZD2018044) and Anhui Provincial Natural Science Foundation (No. 1608085QF151), and the second author is thankful to DST, New Delhi, India, for its financial support under an INSPIRE fellowship, without which this research would not have been possible.

\section{Availability of data and materials}

All of the authors clare that all the data can be accessed in our manuscript in the numerical simulation section.

\section{Competing interests}

All the authors declare that they have no financial and non-financial competing interests.

\section{Authors' contributions}

All authors contributed equally to the writing of this paper. All authors read and approved the final manuscript.

\section{Author details}

'School of Management Science and Engineering, Anhui University of Finance and Economics, Bengbu, China.

${ }^{2}$ Department of Mathematics, National Institute of Technology Durgapur, Durgapur, India.

\section{Publisher's Note}

Springer Nature remains neutral with regard to jurisdictional claims in published maps and institutional affiliations.

Received: 18 May 2018 Accepted: 6 September 2018 Published online: 20 September 2018

\section{References}

1. Kermack, W.O., McKendrick, A.G.: A contribution to the mathematical theory of epidemics. Proc. R. Soc. Lond. A 115(772), 700-721 (1927)

2. Kermack, W.O., McKendrick, A.G.: A contribution to the mathematical theory of epidemics II. The problem of endemicity. Proc. R. Soc. Lond. A 138(834), 55-82 (1932)

3. Billarda, L., Dayananda, P.W.A.: A multi-stage compartmental model for HIV-infected individuals: I waiting time approach. Math. Biosci. 249, 92-101 (2014)

4. Pongsumpun, P., Tang, I.M.: Dynamics of a new strain of the H1N1 influenza a virus incorporating the effects of repetitive contacts. Comput. Math. Methods Med. 2014, Article ID 487974 (2014)

5. Upadhyay, R.K., Kumari, N., Rao, V.S.H.: Modeling the spread of bird flu and predicting outbreak diversity. Nonlinear Anal., Real World Appl. 9, 1638-1648 (2008)

6. Naheed, A., Singh, M., Lucy, D.: Numerical study of SARS epidemic model with the inclusion of diffusion in the system. Appl. Math. Comput. 229, 480-498 (2014) 
7. Kermack, W.O., Mckendrick, A.G.: A contribution to the mathematical theory of epidemics. Proc. R. Soc. A 115(772), 700-721 (1927)

8. Kermack, W.O., Mckendrick, A.G.: Contributions to the mathematical theory of epidemics. II, the problem of endemicity. Proc. R. Soc. A 138(834), 55-83 (1932)

9. Jin, Y., Wang, W., Xiao, S.: An SIRS model with a nonlinear incidence rate. Chaos Solitons Fractals 34, 1482-1497 (2007)

10. Lahrouz, A., Omari, L., Kiouach, D.: Global analysis of a deterministic and stochastic nonlinear SIRS epidemic model. Nonlinear Anal., Model. Control 16, 69-76 (2011)

11. Zhang, T.L., Liu, J.L., Teng, Z.D.: Stability of Hopf bifurcation of a delayed SIRS epidemic model with stage structure Nonlinear Anal., Real World Appl. 11, 293-306 (2010)

12. Zhang, Y.Y., Jia, J.W.: Hopf bifurcation of an epidemic model with a nonlinear birth in population and vertical transmission. Appl. Math. Comput. 230, 164-173 (2014)

13. Xu, R.: Global dynamics of an SElS epidemic model with saturation incidence and latent period. Appl. Math. Comput. 218,7927-7938 (2012)

14. Liu, J.: Bifurcation of a delayed SEIS epidemic model with a changing delitescence and nonlinear incidence rate. Discrete Dyn. Nat. Soc. 2017, Article ID 2340549 (2017)

15. Guo, S.M., Li, X.Z., Song, X.Y.: Stability of an age-structured SEIS epidemic model with infectivity in incubative period. Int. J. Biomath. 3, 299-312 (2010)

16. Yang, B.: Stochastic dynamics of an SEIS epidemic model. Adv. Differ. Equ. 2016, 226 (2016)

17. Witbooi, P.J.: Stability of an SEIR epidemic model with independent stochastic perturbations. Phys. A, Stat. Mech. Appl. 392, 4928-4936 (2013)

18. Yang, Q., Mao, X.: Extinction and recurrence of multi-group SEIR epidemic models with stochastic perturbations. Nonlinear Anal., Real World Appl. 14, 1434-1456 (2013)

19. Zhou, X., Cui, J.: Analysis of stability and bifurcation for a SEIR epidemic model with saturated recovery rate. Commun. Nonlinear Sci. Numer. Simul. 16, 4438-4450 (2011)

20. Liu, L., Wang, J., Liu, X.: Global stability of an SEIR epidemic model with age-dependent latency and relapse. Nonlinear Anal., Real World Appl. 24, 18-35 (2015)

21. Fan, X.L., Wang, L., Teng, Z.D.: Global dynamics for a class of discrete SEIRS epidemic models with general nonlinear incidence. Adv. Differ. Equ. 2013, 123 (2016)

22. Meng, X., Chen, L., Cheng, H.: Two profitless delays for the SEIRS epidemic disease model with nonlinear incidence and pulse vaccination. Appl. Math. Comput. 186, 516-529 (2007)

23. Xu, R., Ma, Z.E.: Global stability of a delayed SEIRS epidemic model with saturation. Nonlinear Dyn. 61, 229-239 (2010)

24. Kuniya, T., Nakata, Y.: Permanence and extinction for a nonautonomous SEIRS epidemic model. Appl. Math. Comput. 218, 9321-9331 (2012)

25. Zhang, L.J., Li, Y.Q., Ren, Q.Q., Huo, Z.X.: Global dynamics of an SEIRS epidemic model with constant immigration and immunity. WSEAS Trans. Math. 5, 630-640 (2013)

26. Gumel, A.B., Moghadas, S.M.: A qualitative study of a vaccination model with non-linear incidence. Appl. Math. Comput. 143, 409-419 (2003)

27. Buonomo, B., Lacitignola, D., Leon, C.V.D.: Qualitative analysis and optimal control of an epidemic model with vaccination and treatment. Math. Comput. Simul. 100, 88-102 (2014)

28. Li, J.Q., Yang, Y.L., Zhou, Y.C.: Global stability of an epidemic model with latent stage and vaccination. Nonlinear Anal., Real World Appl. 12, 2163-2173 (2011)

29. Chen, X.Y., Cao, J.D., Park, J.H., Qiu, J.L.: Stability analysis and estimation of domain of attraction for the endemic equilibrium of an SEIQ epidemic model. Nonlinear Dyn. 87, 975-985 (2017)

30. Li, J., Sun, G.Q., Jin, Z.: Pattern formation of an epidemic model with time delay. Physica A 403, 100-109 (2014)

31. Bai, Z.G., Wu, S.L.: Traveling waves in a delayed SIR epidemic model with nonlinear incidence. Appl. Math. Comput. $263,221-232(2015)$

32. Liu, Q., Chen, Q.M., Jiang, D.Q.: The threshold of a stochastic delayed SIR epidemic model with temporary immunity. Physica A 450, 115-125 (2016)

33. Hu, Z.Y., Chang, L.L., Teng, Z.D., Chen, X.: Bifurcation analysis of a discrete SIRS epidemic model with standard incidence rate. Adv. Differ. Equ. 2016, 155 (2016)

34. Li, J.H., Teng, Z.D.: Bifurcations of an SIRS model with generalized non-monotone incidence rate. Adv. Differ. Equ. $2018,217(2018)$

35. Liu, Q.M., Sun, M.C., Li, T.: Analysis of an SIRS epidemic model with time delay on heterogeneous network. Adv. Differ. Equ. 2017, 309 (2017)

36. Liu, J., Wang, K.: Dynamics of an epidemic model with delays and stage structure. Comput. Appl. Math. 37 2294-2308 (2018)

37. Sharma, N., Gupta, A.K.: Impact of time delay on the dynamics of SEIR epidemic model using cellular automata. Physica A 471, 114-125 (2017)

38. Krishnapriya, P., Pitchaimani, M., Witten, T.M.: Mathematical analysis of an influenza epidemic model with discrete delay. J. Comput. Appl. Math. 324, 155-172 (2017)

39. Jiang, Z.C., Ma, W.B., Wei, J.J.: Global Hopf bifurcation and permanence of a delayed SEIRS epidemic model. Math. Comput. Simul. 122, 35-54 (2016)

40. Liu, J., Wang, K.: Hopf bifurcation of a delayed SIQR epidemic model with constant input and nonlinear incidence rate. Adv. Differ. Equ. 2016, 309 (2016)

41. Fonda, A.: Uniformly persistent semidynamical systems. Proc. Am. Math. Soc. 104, 111-116 (1988)

42. Kundu, S., Maitra, S.: Stability and delay in a three species predator-prey system. AIP Conf. Proc. 1751, 020004 (2016). https://doi.org/10.1063/1.4954857

43. Kundu, S., Maitra, S.: Dynamical behaviour of a delayed three species predator-prey model with cooperation among the prey species. Nonlinear Dyn. 92, 627-643 (2018)

44. Hassard, B.D., Kazarinoff, N.D., Wan, Y.H.: Theory and Applications of Hopf Bifurcation. Cambridge University Press, Cambridge (1981) 
45. Li, X.L., Wei, J.J.: On the zeros of a fourth degree exponential polynomial with applications to a neural network model with delays. Chaos Solitons Fractals 26, 519-526 (2005)

46. Jana, D., Agrawal, R., Upadhyay, R.K.: Top-predator interference and gestation delay as determinants of the dynamics of a realistic model food chain. Chaos Solitons Fractals 69, 50-63 (2014)

Submit your manuscript to a SpringerOpen ${ }^{\circ}$ journal and benefit from:

- Convenient online submission

- Rigorous peer review

- Open access: articles freely available online

- High visibility within the field

- Retaining the copyright to your article

Submit your next manuscript at $\gg$ springeropen.com 